\title{
Somatosensory evoked responses in the diagnosis of thoracic outlet syndrome
}

\author{
C YIANNIKAS, JC WALSH \\ From the Department of Clinical Neurophysiology, Royal Prince Alfred Hospital, Sydney, Australia
}

SUMMARY A study was made of 11 patients with cervical rib, and one patient with Klippel-Fiel syndrome and enlarged transverse processes to determine whether evoked potentials recorded from both Erb's points and the cervical spine in response to median and ulnar nerve stimulation provided information additional to that obtained by EMG and peripheral conduction studies. It was found that in seven patients who had pain and paraesthesias but no objective neurological signs both the peripheral and central conduction studies were within normal limits. By contrast, of five patients who had objective signs, conventional EMG and conduction studies were abnormal in three patients, but abnormalities of the evoked potentials obtained from ulnar nerve stimulation were obtained in all five patients. It is suggested that this application of evoked potential estimation is a useful addition to the more conventional peripheral investigations.

The thoracic outlet syndrome is characterised by a combination of pain in the arm provoked by traction, colour changes in the hand, and a radicular pattern of sensory loss and weakness with intact tendon reflexes. The incidence of thoracic outlet syndrome is uncertain, and the diagnostic criteria are disputed. The abnormalities in motor and sensory conduction in the peripheral segments of the median and ulnar nerves in patients with cervical ribs or bands have been well described by Gilliatt et $a l^{1}$ and Wulff and Gilliatt. ${ }^{2}$ Shahani et $^{2} l^{3}$ Dorfman, ${ }^{4}$ and Eisen $e t a^{5}$ have studied the use of $\mathrm{F}$ wave latency in the diagnosis of thoracic outlet syndrome.

The major abnormalities described in these studies were reduction in amplitudes of the ulnar sensory action potentials, chronic partial denervation in the intrinsic muscles of the involved hand (C8-T1) and prolongation of the $\mathrm{F}$ wave latency. However, in some patients with wasted hands the nerve conduction studies were normal and if there were no associated objective signs the diagnostic yield from electrophysiological studies was low. ${ }^{4}$ The difficulties that arise in the electrophysiological diagnosis are related to the problem of assessing nerve conduction across the brachial plexus. Some

Address for reprint requests: Dr J Walsh, Dept of Clin Neurophysiology, Royal Prince Alfred Hospital, Sydney, N.S.W. 2050 Australia.

Received 24 May 1982. Accepted 31 August 1982. workers have used transclavicular nerve conduction studies in an attempt to study conduction across the plexus but the results of such studies have been conflicting. ${ }^{6-8}$

The lack of an objective method for diagnosing thoracic outlet syndrome and monitoring the results of treatment has prevented a clear understanding of its natural history and of the indications for surgery. Somatosensory studies with surface recordings from the region of Erb's point, cervical spine, and somatosensory cortex have been used in the study of patients with brachial plexus traction lesions ${ }^{9}$ and cervical spondylosis ${ }^{1011}$, but the place of these techniques in the thoracic outlet syndrome has not been fully assessed. Siivola $e$ al $^{12}$ studied one case of costo-clavicular syndrome and found an absent response from Erb's point on ulnar nerve stimulation at the wrist and the elbow with normal responses from median nerve stimulation. Glover et al $^{13}$ found a high incidence of abnormalities in their cases of thoracic outlet syndrome. However, the clinical criteria for diagnosis and the nature of the evoked potential abnormalities were not discussed. Ioyne and Buchthal ${ }^{14}$ have used root recording techniques in a small group of patients with cervical ribs but the technique does not appear suitable for routine diagnostic use.

The purpose of this study was to evaluate the diagnostic usefulness of somatosensory evoked potentials in a group of patients with radiologically proven cervical ribs or enlarged transverse processes 
of the cervical spine who had symptoms and signs consistent with thoracic outlet syndrome.

\section{Patients and methods}

Twelve patients were studied, eight females and four males. The ages ranged from 5 to 50 years with a mean age of 30.9 and a median age of 34 years. All patients submitted to the study had radiologically proven cervical ribs, or in one case Klippel-Fiel syndrome with enlarged transverse processes. The patients were classified into two groups according to the nature of their clinical condition.

Group 1 consisted of seven patients with radiating pain or paraesthesias in the arm and fingers without any objective neurological signs.

Group 2 consisted of five patients who had objective neurological signs such as loss of sensation, weakness, or wasting of the small muscles of the hand. In two cases there were objective features of vascular compression.

All patients had motor and sensory conduction studies performed on the median and ulnar nerves of both upper limbs and electromyography was performed on the relevant muscles. The control studies were performed on 11 persons with no evidence of any disorder of the arm or neck. Somatosensory evoked potentials (SEPs) were performed using a similar technique to that previously described by Jones. ${ }^{15}$ Recording was performed using subdermal needle electrodes overlying Erb's point and the upper cervical vertebrae (Cv2), with a common midfrontal reference electrode (Fz: 10-20 system). The stimulus was a square wave of $100 \mu$ s duration delivered to the skin with the cathode overlying the median or ulnar nerves at the wrist and proximal to the anode. The stimulus strength was adjusted to produce a twitch of the appropriate muscles. The stimuli were delivered at a rate of $2 \mathrm{~Hz}$ and could be tolerated for long periods without discomfort. Subjects sat in an armchair and were instructed to relax and to sleep if they wished. The recording electrodes were connected to the inputs of Medelec PA62 preamplifiers and fed into Medelec AA6MKIII main amplifiers. The input impedence of the system was $100 \mathrm{M} \Omega$. The bandpass used was $16 \mathrm{~Hz}-3 \cdot 2 \mathrm{kHz}$. The averaged response of 256 stimuli was obtained using a DAV6 digital averaging system with an analysis time of $50 \mathrm{~ms}$ giving an A-D sampling rate of 20 points $/ \mathrm{ms}$. The signal latencies were determined from photographic records and were measured from the stimulus artefact to the peak negativity of the responses.
The amplitudes were measured from the onset of the response to the peak negativity. In this study the terms N9 and $\mathrm{N} 13$ will be used to denote the mean latency of the negative peaks of the responses from Erb's point and cervical spine respectively. The conduction times (CT) as measured between peak latencies will be denoted N13-N9.

\section{Results}

\section{NORMAL SOMATOSENSORY EVOKED \\ POTENTIALS}

The mean and standard deviation of the amplitude and latency of each potential for the control subjects, together with the differences encountered between left and right arm stimulation are given in table 1. In each individual the left/right amplitude difference for a given potential, expressed as a percentage of the amplitude on the side with the largest response, was less than $50 \%$ (N9) and $49 \%$ (N13) for the ulnar nerve and $54 \%$ (N9), $40 \%$ (N13) for median nerve stimulation. The maximal individual left/right latency difference for each potential were less than $0.9 \mathrm{~ms}(\mathrm{~N} 9), 0.5$ (N13) for the ulnar and 0.8 (N13) for the median nerve. Figure 1 illustrates a normal Erb's and cervical spinal response produced on stimulation of the median and ulnar nerves.

\section{SOMATOSENSORY EVOKED POTENTIALS IN THE PATIENT GROUP}

Group 1 This group consisted of seven patients with radicular pain and paraesthesias as the main symptoms. The mean SEP results and standard deviation of this group are illustrated in table 2 . The amplitudes and latencies of the N9 and $\mathrm{N} 13$ and the conduction times on both sides were within normal limits. There was no significant difference in amplitude and latency between the symptomatic (S) and asymptomatic (A) sides. The nerve conduction studies and EMG were within normal limits.

Group 2 This group consisted of five patients with radicular pain and paraesthesias and objective signs on neurological examination. The peripheral electrophysiological studies in three cases demonstrated

Table 1 The mean values for the amplitude, latency, conduction times (mean $\pm S D$ ), maximal $L-R$ difference and the median ulnar latency difference of evoked potentials in 11 normal subjects on stimulating the median and ulnar nerves at the wrist.

\begin{tabular}{|c|c|c|c|c|c|c|c|c|c|}
\hline & \multicolumn{4}{|l|}{ Median } & \multicolumn{4}{|l|}{ Ulnar } & \multirow{2}{*}{$\begin{array}{l}\begin{array}{l}\text { Median ulnar } \\
\text { difference }\end{array} \\
\text { Latency }(\mathrm{ms}) \\
\text { mean } \pm S D\end{array}$} \\
\hline & $\begin{array}{l}\text { Latency (ms) } \\
\text { mean } \pm S D\end{array}$ & $\begin{array}{l}\text { Amplitude }(u V) \\
\text { mean } \pm S D\end{array}$ & $\frac{\text { Maximal }}{\text { lat. }(m s)}$ & $\frac{-R \text { diff. }}{a m p}(\%)$ & $\begin{array}{l}\text { Latency }(\mathrm{ms}) \\
\text { mean } \pm S D\end{array}$ & $\begin{array}{l}\text { Amplitude }(u V) \\
\text { mean } \pm S D\end{array}$ & $\frac{\text { Maximal }}{\text { lat. }(\mathrm{ms})}$ & $\frac{-R \text { diff. }}{a m p(\%)}$ & \\
\hline $\begin{array}{l}\text { N9 } \\
\text { N13 } \\
\text { N13-9 }\end{array}$ & $\begin{aligned} 9.9 & \pm 0.7 \\
13.4 & \pm 0.6 \\
3.5 & \pm 0.5\end{aligned}$ & $\begin{array}{l}7 \cdot 3 \pm 2.2 \\
3.9 \pm 0.8\end{array}$ & $\begin{array}{l}0 \cdot 8 \\
0 \cdot 8 \\
0 \cdot 5\end{array}$ & $\begin{array}{l}54 \\
40\end{array}$ & $\begin{array}{r}10 \cdot 5 \pm 0.9 \\
14 \cdot 3 \pm 1 \cdot 2 \\
3 \cdot 9 \pm 0.5\end{array}$ & $\begin{array}{l}3.4 \pm 1.4 \\
2.1 \pm 0.7\end{array}$ & $\begin{array}{l}0.9 \\
0.5 \\
0.8\end{array}$ & $\begin{array}{l}50 \\
49\end{array}$ & $\begin{array}{l}0 \cdot 6 \pm 0 \cdot 3 \\
1 \cdot 0 \pm 0 \cdot 5\end{array}$ \\
\hline
\end{tabular}

Arm Length mean $-71.8 \mathrm{~cm}$. 


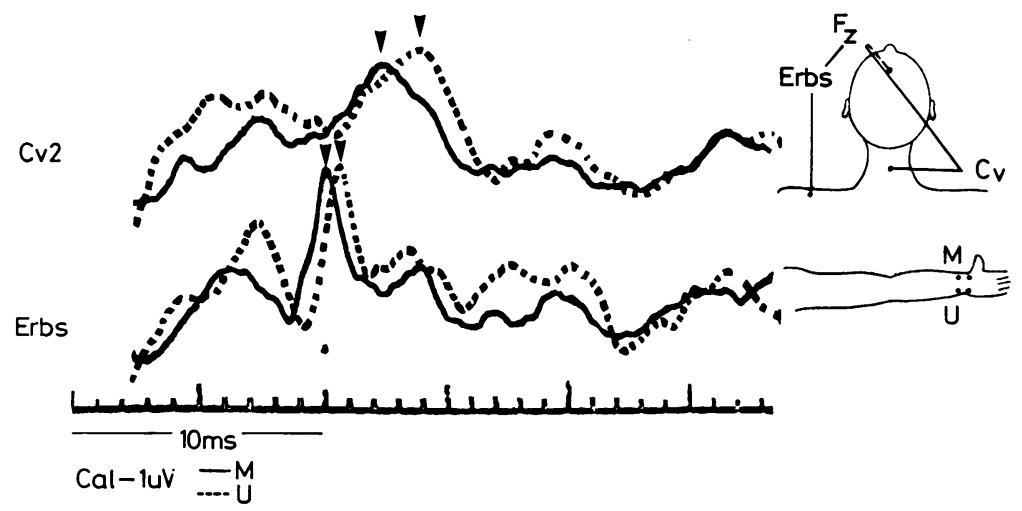

Fig 1 Somatosensory evoked potentials simultaneously recorded from 2 electrode locations with a mid-frontal reference on stimulation of the median $(M)$ and ulnar $(U)$ nerves at the wrist of a normal subject. The electrodes were located over the 2nd cervical vertebrae $(\mathrm{Cv} 2)$ and ipsilateral Erb's point.

the classical changes described by Gilliatt $e t$ al ${ }^{1}$ with denervation in the small muscles of the hand, normal conduction velocities and reduction in amplitude of the ulnar sensory action potential. Two cases had normal conduction studies. The SEP results are illustrated in table 3 . The N9, N13 latencies, N13-N9 conduction time and the N9 and N13 amplitudes on stimulating the median nerve were within normal limits, as were the maximal individual bilateral latency and amplitude difference. On ulnar nerve stimulation there was a significant prolongation of the N9, N13 latencies, N9-N13 conduction time and median-ulnar latency difference in comparison to the results from the less symptomatic side. The asymptomatic to symptomatic difference in latency and amplitudes was significantly abnormal when compared to the control group left-right difference. Useful comparison of absolute latencies with the control group was not possible because of significantly shorter arm lengths in the patient group. The maximum individual bilateral amplitude difference was greater than $60 \%$ for $\mathrm{N} 9$ and in two cases the N13 response was absent. Two types of abnormalities were seen on stimulating the ulnar

Table 2 The mean values for the amplitude, latency, conduction times (mean $\pm S D$ ), the maximal asymptomatic (A) symptomatic $(S)$ difference and the median-ulnar latency difference of evoked potentials in 7 patients (Group I) obtained on stimulating the median and ulnar nerves at the wrist.

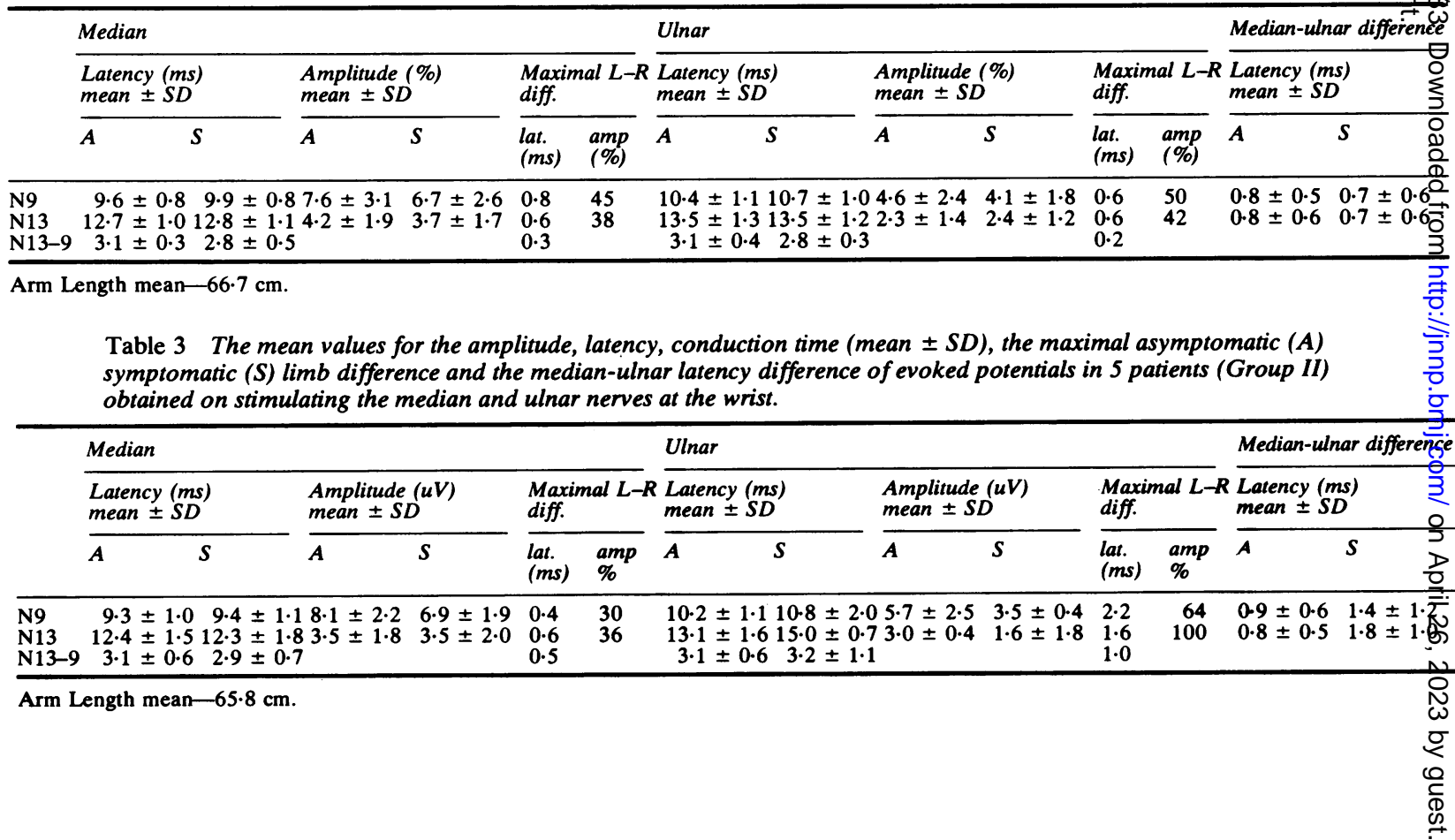




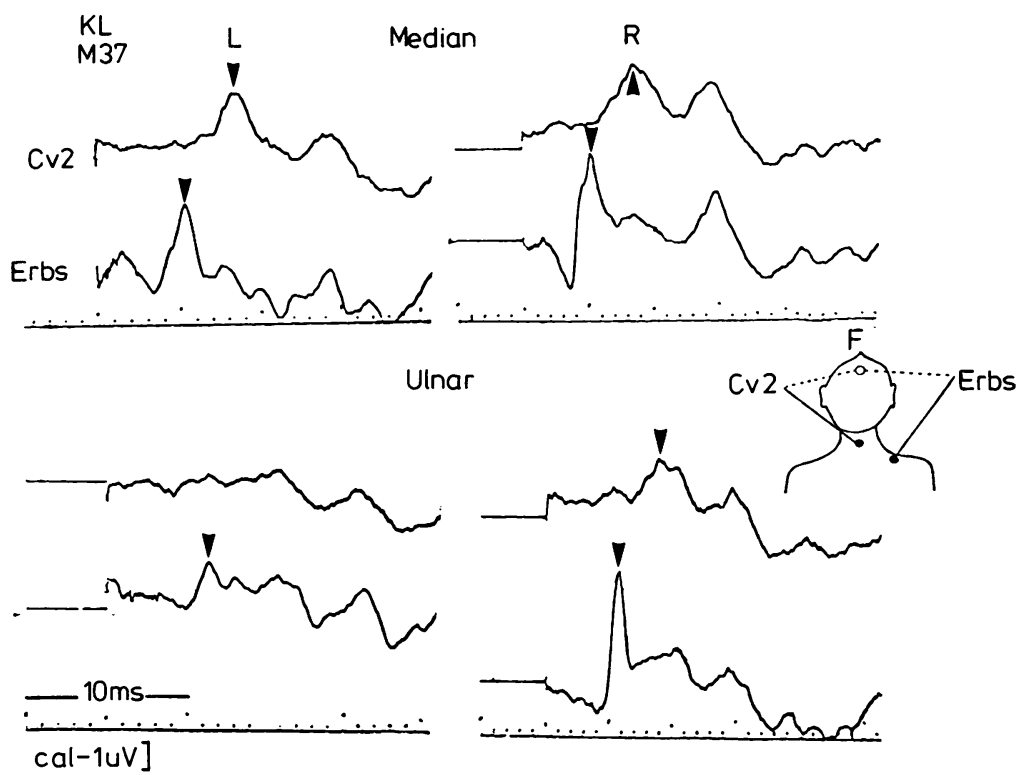

Case A

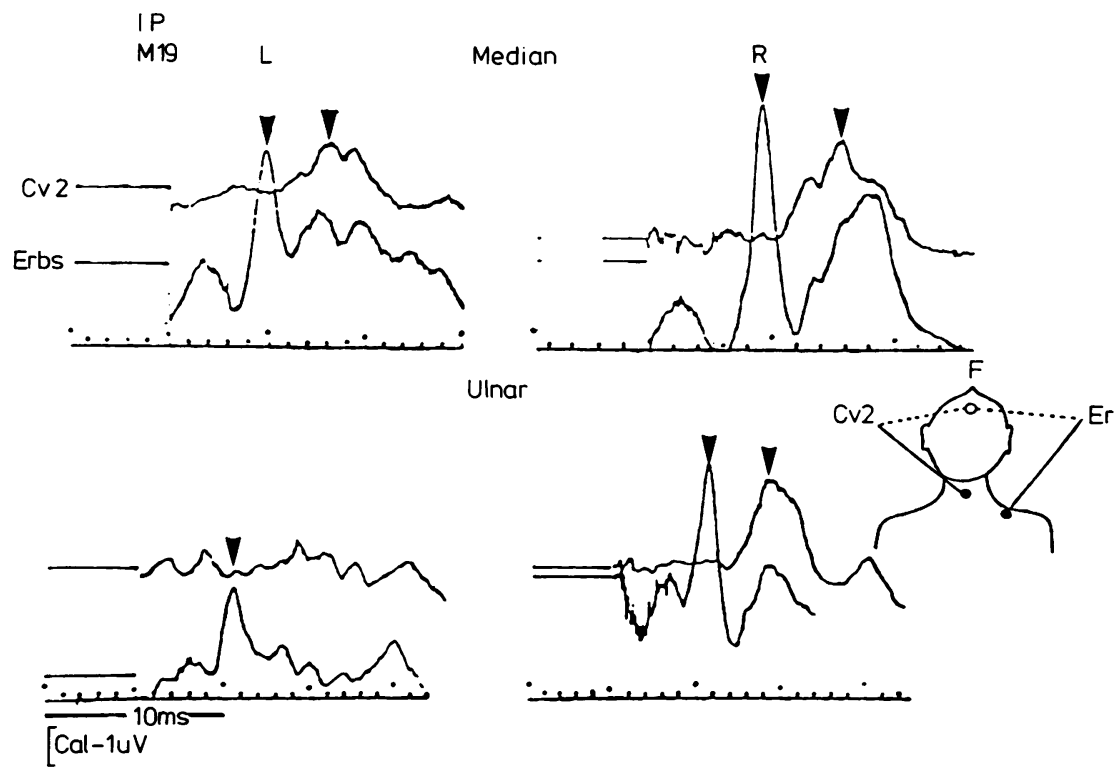

Case B

Fig 2 Somatosensory evoked potentials on median and ulnar nerve stimulation in 2 patients from group II. The responses on stimulation of the asymptomatic side are shown for comparison. On stimulation of the ulnar nerve, the N13 is absent for the affected left arm in case $A$ and is of low amplitude for the affected right arm in case $B$. The amplitude of the $N 9$ response is reduced in case $A$ and normal in case $B$. The $N 9$ latencies are normal. 
nerve: the first, as demonstrated by the following two cases, was a relatively normal N9 response with an absent or low amplitude N13 response. Figure 2(b) illustrates the results in a 19-yr-old male with Klippel-Fiel disease. The patient complained of paraesthesias in the left hand and on neurological examination demonstrated impaired sensation in the C7-8 dermatomes. There was no evidence of weakness or wasting of the small muscles of the hand. There was also evidence of vascular compression which was confirmed by arteriography. Nerve conduction studies and EMG were normal. The SEP on left ulnar nerve stimulation demonstrated a normal latency low amplitude N9 response and an absent N13 response. Figure 3 illustrates records from a 5 -yr-old female with bilateral cervical ribs and hemi-vertebrae at C7-T2. Neurological examination revealed wasting of the thenar eminence and weakness of abductor pollicus brevis, but sensory examination was normal. Electrophysiological studies demonstrated the presence of denervation in the abductor pollicus brevis and a reduction in amplitude of the ulnar sensory potential. The SEP on ulnar nerve stimulation demonstrated a normal N9 response with a poorly formed N13 response. The responses on stimulating the median nerve were normal in both cases.

The second type of abnormality found on stimulating the ulnar nerve was a reduction in the amplitude and prolongation of the latency of the N9 response, prolonged N13-N9 conduction time, and relatively less impairment of the $\mathrm{N} 13$ response. This is illustrated by the following case. Figure 2(a) illustrates the response from a 37-yr-old male with a left-sided cervical rib, and large transverse processes on C7. The patient complained of bilateral paraesthesias in both arms and in the left hand; on neurological examination there was sensory impairment over the C5 dermatome on both sides and in a C8-T1 distribution in the left hand. There was no evidence of weakness or wasting. Nerve conduction studies and EMG were normal. The SEPs demonstrated a prolongation of the latency and reduction in the amplitude of the $\mathrm{N} 9$ response. The N13-N9 conduction time was prolonged and the N13 amplitude was affected to a lesser extent. In the other 2 cases, one demonstrated a low amplitude N9 response with an increased N13-N9 conduction time and the last case a low amplitude prolonged latency N9 response.

Since four of the five cases had bilateral radiological abnormalities it is interesting that the studies from the less symptomatic or asymptomatic side were similar to the control studies.

\section{Discussion}

Neurovascular compression at the base of the neck has been observed by anatomists and surgeons for over a century. Much controversy existed over the compressive mechanism involved and numerous terms such as scalenus anticus syndrome and shoul-

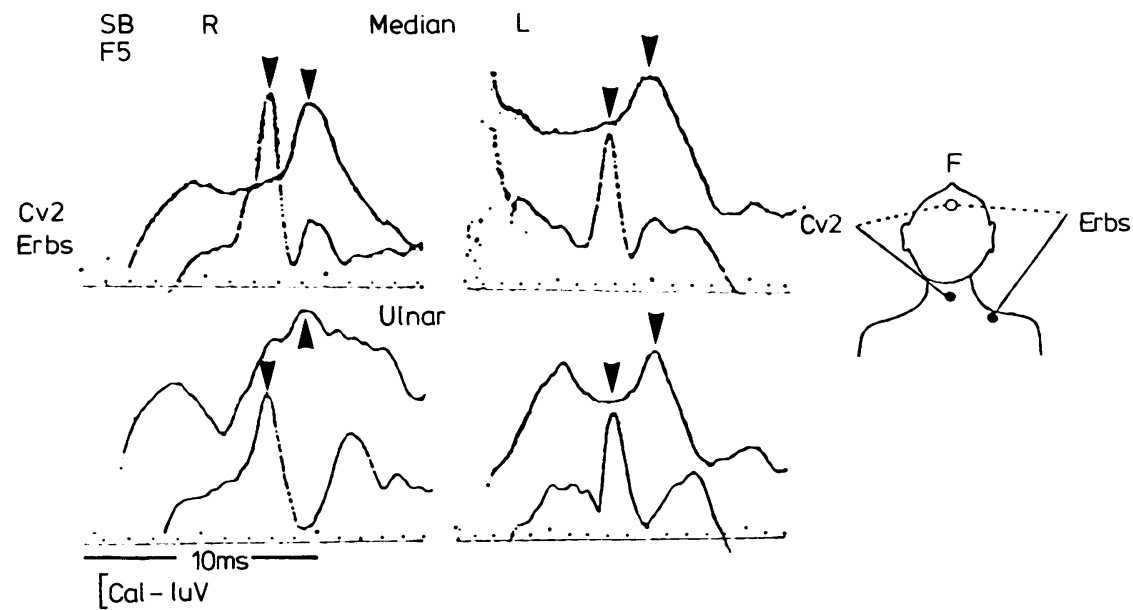

Fig 3 Somatosensory evoked potentials from median and ulnar nerve stimulation in a patient from group II. The responses on stimulating the asymptomatic left arm are shown for comparison. When stimulating the ulnar nerve on the symptomatic side the amplitude of the $N 9$ and $N 13$ responses are reduced, the latency of the $N 9$ response is prolonged and the N13-N9 conduction time is increased. 
der arm syndrome were used, all implicating different anatomical structures as possible causes of this syndrome. In recent years the controversy has been centred on the development of objective parameters to determine the value of surgical intervention.

The neurological aspects of the thoracic outlet syndrome have been clarified by the work of Gilliatt et al $^{16}$ and Lascelles et al. ${ }^{17}$ The use of peripheral nerve conduction and $F$ waves in the assessment of these patients has been well delineated by Gilliatt et $a l^{12}$ and by other workers. In the presence of wasting and weakness there was consistent reduction in the amplitude of the ulnar sensory potential and a prolongation of the $F$ wave latency. The $F$ wave abnormalities did not localise the level of the lesion and these features may be consistent with partial denervation and loss of some large motor fibres. In the absence of weakness or wasting the peripheral nerve conduction studies were normal and in the absence of objective neurological abnormalities neither the nerve conduction nor $F$ wave studies revealed any abnormalities. Attempts have been made to study conduction across the plexus using motor nerve stimulation above the clavicle, however, the results have been contradictory. ${ }^{6-8}$

Although relatively few patients were examined with the present technique, the data suggest that the two clinical groups of patients with thoracic outlet syndrome were associated with characteristic SEP results.

In the first group with no objective neurological signs, the SEP amplitude, latencies and conduction times were normal. These results are consistent with the normal propagation of impulses up the large diameter myelinated Ia and II fibres which are thought to propagate the impulses that generate the N9 and N13 responses. The normal responses indicate that the patients' symptoms arise from irritation of otherwise normal large afferent fibres or of smaller fibres; and that no axonal loss or conduction block involving large diameter has occurred. Such functional disturbances may be due to electrical interaction between adjacent fibres leading to excitation of quiescent neighbouring fibres, or fibre interaction in injured nerves leading to artificial synapses between motor, sensory, and sympathetic fibres. ${ }^{18} 19$ Another possibility is retrograde degeneration of small spinal ganglion cells leading to abnormal activity at a spinal cord level..$^{20}$ Such slowly conducting fibres would not be tested by the present technique. Similar results were obtained by Ioyne and Buchthal ${ }^{14}$ who studied three patients with thoracic outlet syndrome and found abnormalities only in the one case with objective neurological dysfunction.

The second patient group with both symptoms and objective neurological disturbances demonstrated two distinct types of abnormality on stimulating the ulnar nerve. In two patients the N9 component was present and of normal latency with an abnormal or absent N13 response. The N9 potential which is recorded over Erb's point reflects the propagation of a mixed action potential through the brachial plexus, and the N13 potential is derived from the cervical cord or low brainstem. The loss of the N13 response with preservation of the N9 potential could result from a proximal lesion on either side of the dorsal root ganglion. The N9 response may then be attenuated due to secondary degeneration of distal plexus fibres if the lesion was distal to the ganglia. In the other three cases the SEP showed an attenuation and prolongation of the latency of the N9 component with the N13 affected to an equal or lesser extent and/or an increase in the N13-N9 conduction time. This suggests a distal lesion leading to involvement of large diameter fibres in the lower plexus.

It is interesting to note that all of the patients in group I, and two of the patients in group II, had normal EMG and peripheral conduction studies. The two patients in group II with normal peripheral studies had no weakness or wasting but had objective sensory disturbances, and in these cases the sensitivity of the SEP, when both the median and ulnar nerves were stimulated, was greater than that of peripheral studies.

The present study indicates that the somatosensory evoked potentials recorded from the brachial plexus and cervical spine can yield valuable diagnostic information in patients with thoracic outlet syndrome and add greater sensitivity to the conventional electrophysiological studies if more than one nerve is studied. The method, in combination with conventional peripheral studies can differentiate between distal entrapment lesions, plexus lesions and lesions in more proximal structures. Furthermore, the studies may be of assistance in deciding which patients would benefit from surgical intervention.

\section{References}

${ }^{1}$ Gilliatt RW, Willison RG, Dietz V, Williams IR. Peripheral nerve conduction in patients with a cervical rib or band. Ann Neurol 1978;4:124-9.

${ }^{2}$ Wulff $\mathrm{CH}$, Gilliatt RW. F waves in patients with hand wasting caused by a cervical rib or band. Muscle Nerve 1979;2:452-7.

${ }^{3}$ Shahani BT, Potts F, Juguilon A, Young RR. Electrophysiological studies in thoracic outlet syndrome. Muscle Nerve 1980;3:182.

${ }^{4}$ Dorfman LJ. F wave latency in the cervical rib and band syndrome. Muscle Nerve 1979;2:158. 
${ }^{5}$ Eisen A, Schomer D, Melmed C. The application of F wave measurements in the differentiation of proximal and distal upper limb entrapments. Neurology (Minneap) 1977;27:662-8.

${ }^{6}$ Daube JR. Nerve conduction studies in thoracic outlet syndrome. Neurology (Minneap) 1975;25:347.

${ }^{7}$ Urschel Jr, HC, Paulson DL, McNamara JJ. Thoracic outlet syndrome. Ann Thoracic Surg 1968;6:1-10.

${ }^{8}$ Kremer RM, Ahlquist Jr, RE. Thoracic outlet syndrome. Am J Surg 1975;130:612-16.

9 Jones SJ. Investigation of brachial plexus traction lesions by peripheral and spinal somatosensory evoked potentials. J Neurol Neurosurg Psychiatry 1979;42:107-16.

${ }^{10} \mathrm{El}$ Negamy E, Sedgwick EM. Delayed cervical somatosensory potentials in cervical spondylosis. $J$ Neurol Neurosurg Psychiatry 1979;42:238-41.

"Ganes T. Somatosensory conduction times and peripheral, cervical and cortical evoked potentials in patients with cervical spondylosis. J Neurol Neurosurg Psychiatry 1980;43:683-9.

${ }^{12}$ Siivola J, Myllylä VV, Sulg I, Hokkanen E. Brachial plexus and radicular neurography in relation to cortical evoked responses. J Neurol Neurosurg Psychiatry 1979;42:1151-8.
${ }^{13}$ Glover JL, Worth RM, Bendick BJ, Hall PV, Morkand ' DM. Evoked responses in the diagnosis of thoracic outlet syndrome. Surgery 1981;89:82-8.

${ }^{14}$ Inouye I, Buchthal F. Segmental sensory innervation determined by potentials recorded from cervical spinal nerves. Brain 1977;100:731-48.

15 Jones SJ. Short latency potentials recorded from the neck and scalp following median nerve stimulation in man. Electroencephalogr Clin Neurophys 1977;43:853-63.

${ }^{16}$ Gilliatt RW, Le Quesne PM, Logue V, Sumner AJ. Wasting of the hand associated with cervical rib or band. J Neurol Neurosurg Psychiatry 1970;33:61524.

${ }^{17}$ Lascelles RG, Mohr PD, Neary D, Bloor K. The thoracic outlet syndrome. Brain 1977;100:601-12.

${ }^{18}$ Katz B, Schmitt DH. Electric interaction between two adjacent nerve fibres. J Physiol (Lond) 1940;97:471-88.

${ }^{19}$ Granit R, Leksell L, Skoglund CR. Fibre interaction in injured or compressed region of nerve. Brain 1944;67:125-40.

${ }^{20}$ Sunderland S. Pain mechanisms in causalgia. J Neurol Neurosurg Psychiatry 1976;39:471-80. 